\title{
Beyond Horndeski interactions induced by quantum effects
}

\author{
B. Latosh *1,2 \\ ${ }^{1}$ Bogoliubov Laboratory of Theoretical Physics, JINR, Dubna 141980, Russia \\ ${ }^{2}$ Dubna State University, Universitetskaya str. 19, Dubna 141982, Russia
}

\begin{abstract}
Opportunity to generate beyond Horndeski interactions is addressed. An amplitude generating a certain beyond Horndeski coupling is explicitly found. The amplitude is free from ultraviolet divergences, so it is protected from ultraviolet contributions and can be considered as a universal prediction of effective field theory.
\end{abstract}

\section{Introduction}

Horndeski models are scalar-tensor models that have second-order field equations and have no interaction between the scalar field and matter [1, 2]. Because of the second-order field equations they are protected from the Ostrogradsky instability associated with higher derivatives [3]. The Horndeski action reads (Jordan frame):

$$
\begin{aligned}
& \mathcal{A}=\int d^{4} x \sqrt{-g}\left[\mathcal{L}_{2}+\mathcal{L}_{3}+\mathcal{L}_{4}+\mathcal{L}_{5}+\mathcal{L}_{\text {matter }}\left[\Psi, g_{\mu \nu}\right]\right], \\
\mathcal{L}_{2}= & G_{2}, \\
\mathcal{L}_{3}= & G_{3} \square \phi \\
\mathcal{L}_{4}= & G_{4} R+G_{4, X}\left[(\square \phi)^{2}-\left(\nabla_{\mu \nu} \phi\right)^{2}\right] \\
\mathcal{L}_{5}= & G_{5} G^{\mu \nu} \nabla_{\mu \nu} \phi-\frac{1}{6} G_{5, X}\left[(\square \phi)^{3}-3\left(\nabla_{\mu \nu} \phi\right)^{2} \square \phi+2\left(\nabla_{\mu \nu} \phi\right)^{3}\right]
\end{aligned}
$$

Here $\Psi$ notes matter degrees of freedom; $G_{i}$ are arbitrary functions of the scalar field $\phi$ and its canonical kinetic term $X=1 / 2(\partial \phi)^{2} ; G_{i, X}$ are derivatives with respect to $X ; R$ is the Ricci scalar, and $G_{\mu \nu}$ is the Einstein tensor.

Beyond Horndeski models generalize Horndeski gravity. These models introduce a nonminimal coupling between the scalar field and matter which preserves the second differential order of field equations. The most general beyond Horndeski non-minimal coupling is described by the following Lagrangian [4]:

$$
\mathcal{A}_{\mathrm{int}}=\int d^{4} x \sqrt{-g}\left[C(\phi, X) g_{\mu \nu}+D(\phi, X) \partial_{\mu} \phi \partial_{\nu} \phi\right] T^{\mu \nu}
$$

Here $C$ and $D$ are arbitrary functions of the scalar field $\phi$ and the canonical kinetic term $X$.

It must be noted that both Horndeski and beyond Horndeski models are well motivated and widely studied [5, 6, 7, 8, 9, 10, 11]. In particular, beyond Horndeski models originated from degenerate higher-order scalar-tensor (DHOST) theories [12, 13, 14, 15, 16, 17, 18, 19] and it was shown that only models (3) have no ghost modes in the spectrum of tensor perturbations propagating about a cosmological background [18, 19]. More detailed reviews of these models can be found elsewhere [4, 20].

Within modified gravity the usage of Horndeski and beyond Horndeski models is well-motivated, as these models, in some sense, describe the most general class of physically acceptable models. In this paper we argue that some beyond Horndeski interactions are generated dynamically within quantum theory.

*latosh@theor.jinr.ru 
Quantum behavior of a gravity theory can be consistently described within effective field theory formalism $[21,22,23]$. Such an approach to gravity is well studied for general relativity and it allows one to obtain some verifiable predictions [24, 25, 26]. We also should note that one should not neglect the whole manifold of alternative approaches to quantum features of scalar-tensor theories [27, 28, 29, 30, 31, 32, 33, 34].

Recently it was found that a certain non-minimal interaction between the scalar field and gravity is dynamically induced at the one-loop level [26]. The effect is a complete analogy with the anomalous fermion dipole moment. In this paper we show that this non-minimal interaction is also responsible for a dynamical generation of a certain beyond Horndeski interaction. We also show that some other beyond Horndeski interactions can be generated at the loop level, although they will be strongly suppressed.

This paper is organized as follows. In Section 2 we briefly discuss the effective field theory method, its implementation to gravity, and the mechanism responsible for the generation of a new non-minimal coupling between the scalar field and gravity. In Section 3 we show that the same mechanism generates certain beyond Horndeski interactions at the one-loop level. We discuss what interactions can be generated by such a mechanism and point to the fact that some of them are suppressed more then the others. We highlight the interaction which provides the leading contribution in the low energy regime. We conclude in Section 4 where we discuss the physical role of such interactions.

\section{Anomalous non-minimal coupling}

Effective field theory method originated within particle physics [35] and recently was applied to gravity $[21,22,23]$. Its main premise is the factorization of ultraviolet physics. In the context of gravity this means that low energy phenomena, for instance, Solar system physics, are not affected by the Planck scale fluctuations in a meaningful way. This allows one to set a factorization scale ${ }^{1} \mu$, which is naturally should lie below the Planck scale, and to construct a low energy theory as a momentum expansion. This construction factorizes out all phenomena lying above the factorization scale $\mu$ and provides a consistent quantum description of the low energy physics.

On the practical ground the effective field theory paradigm is implemented to gravity as follows. Firstly, one sets the factorization scale $\mu$ which lies below the Planck mass. Secondly, one defines the microscopic action $\mathcal{A}$ of a gravity theory. The action describes both gravitation and non-gravitational degrees of freedom. Finally, one performs a background field quantization $[37,38,39,40]$.

Background field quantization, in turn, is perform as follows. Firstly, one finds a suitable background metric $\bar{g}_{\mu \nu}$ which solves the classical field equations. Then, one introduces the full metric $g_{\mu \nu}$ which accounts both for the background contribution and for the perturbations propagating about it:

$$
g_{\mu \nu}=\bar{g}_{\mu \nu}+\kappa h_{\mu \nu}
$$

Here $\kappa$ is a dimensional parameter $\left(\kappa^{2}=32 \pi G\right.$ in our case) and $h_{\mu \nu}$ is a field with the canonical mass-dimension which describes small metric perturbations. Lastly, the action $\mathcal{A}$ is expanded in a series with respect to $h_{\mu \nu}$, which is equivalent to an expansion with respect to $\square / m_{P}^{2}$ :

$$
\mathcal{A}[g]=\mathcal{A}[\bar{g}]+\frac{\delta \mathcal{A}}{\delta g_{\mu \nu}} \kappa h_{\mu \nu}+\frac{1}{2} \frac{\delta \mathcal{A}}{\delta g_{\mu \nu} \delta g_{\alpha \beta}} \kappa^{2} h_{\mu \nu} h_{\alpha \beta}+\frac{1}{3 !} \frac{\delta \mathcal{A}}{\delta g_{\mu \nu} \delta g_{\alpha \beta} \delta g_{\rho \sigma}} \kappa^{3} h_{\mu \nu}+O\left(\kappa^{4}\right) .
$$

After that the field $h_{\mu \nu}$ is quantized. In this expansion $\delta^{2} A /\left(\delta g_{\mu \nu} \delta g_{\alpha \beta}\right)$ terms describe propagation of a free graviton, while other terms describe gravitational field self-interaction and interaction with matter.

Effective field approach to gravity is discussed in great details in papers [22, 21], so we will not discuss it further. We would only highlight two things. The first one is the gauge fixing. We use the Fock gauge $\partial_{\mu} h^{\mu \nu}-\frac{1}{2} \partial^{\nu} h=0$ and fix it with the following gauge-fixing term:

$$
\mathcal{L}_{\mathrm{gf}}=\left(\partial_{\mu} h^{\mu \nu}-\frac{1}{2} \partial^{\nu} h\right)^{2}
$$

\footnotetext{
${ }^{1}$ In the previous papers $[26,36]$ is was called "the renormalization scale". Such a terminology is misleading and we will not use it hereafter.
} 
The second one is that we use the flat metric $\eta_{\mu \nu}$ as the background.

An important milestone of effective gravity was found in paper [21] where it was shown that one can use effective theory paradigm to evaluate classical corrections to the Newton potential. Such calculations can be performed due to the structure of loop corrections. The power-law corrections of the Newton potential are given by non-analytical functions in the momentum space [21]:

$$
\begin{aligned}
& \int \frac{d^{3} \vec{k}}{(2 \pi)^{3}} \frac{1}{k} e^{-i \vec{k} \cdot \vec{r}}=\frac{1}{2 \pi^{2} r^{2}} \\
& \int \frac{d^{3} \vec{k}}{(2 \pi)^{3}} \ln (\vec{k})^{2} e^{-i \vec{k} \cdot \vec{r}}=-\frac{1}{2 \pi^{2} r^{3}}
\end{aligned}
$$

Such non-analytical terms are typically generated by loop corrections. In the low energy regime, where we perform the most part of terrestrial experiments, these terms dominate over local corrections. But most importantly, such terms are multiplied by finite coefficients and do not depend on the factorization scale. To put it otherwise, these terms are protected from the high energy content of a given model and can be treated as a universal prediction.

A similar effect was found in paper [26]. In an analogy with [21] the paper addresses an effect that does not depend on the factorization scale. At the one-loop level the following non-minimal interaction between a scalar field and gravity is generated:

$$
\mathcal{L}=G^{\mu \nu} \nabla_{\mu} \phi \nabla_{\nu} \phi
$$

This interaction is generated by the following diagram:

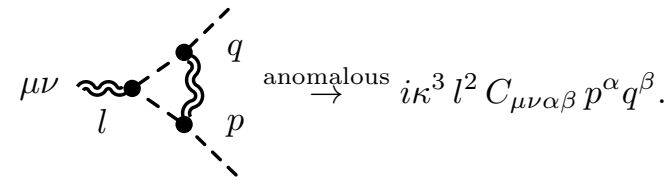

The amplitude contains a contribution free from ultraviolet divergences. Infrared divergences of such an amplitude are regularized via soft graviton radiation. Therefore, in full analogy with the previous case, one finds a contribution that is independent on the high energy content of a model. Moreover, exactly the same phenomenon is well-known within particle physics where it is responsible for the generation of anomalous magnetic momenta.

This provides ground to claim that the non-minimal kinetic interaction (8) is a universal prediction of quantum gravity which is independent on the high energy content of a model. It also should be noted that, unlike the other case [21], such an interaction is local and strongly suppressed. Because of this one can establish some empirical constraints on its coupling [26].

\section{Anomalous beyond Horndeski couplings}

The same mechanism that generates the non-minimal kinetic coupling (8) is responsible for a generation of a certain beyond Horndeski interaction. Let us consider a minimal scalar-tensor model which contains no non-minimal couplings

$$
\mathcal{A}=\int d^{4} x \sqrt{-g}\left[-\frac{1}{16 \pi G} R+\frac{1}{2} g^{\mu \nu} \nabla_{\mu} \phi \nabla_{\nu} \phi+\mathcal{L}_{\text {matter }}\left[\Psi, g_{\mu \nu}\right]\right]
$$

At the one-loop level the model generates the desired non-minimal kinetic coupling [26]. To show that beyond Horndeski interactions are generated in a way similar to the non-minimal coupling we find the specific one-loop amplitude that generates a beyond Horndeski interaction.

Beyond Horndeski interactions deal with a non-minimal interaction between the scalar field and matter (3). Therefore one should study gravitational scattering of the scalar field on matter degrees of freedom. The simplest suitable amplitude corresponds to the following diagram

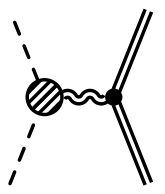


Here we note matter degrees of freedom with a double plain line. We only consider tree-level interaction between the matter and gravity because of the two reasons. Firstly, such corrections lie beyond the scope of this paper and were extensively studied before [21, 24, 41, 42]. Secondly, we are interested in the coupling between regular matter and the new scalar field. Corrections to a coupling between matter and gravity can only alter a coupling between the matter energytensor and gravity. Therefore they are irrelevant for our purpose. Finally, such corrections will introduce an additional factors $\kappa$, so the resulted amplitude receives additional suppression.

Because of this only corrections to the scalar-tensor sector are relevant. Such corrections were discussed in paper [26] and, as we have highlighted, it was shown that an anomalous interaction (9) is generated. This exact anomalous contribution generates an anomalous interaction between matter and the scalar field:

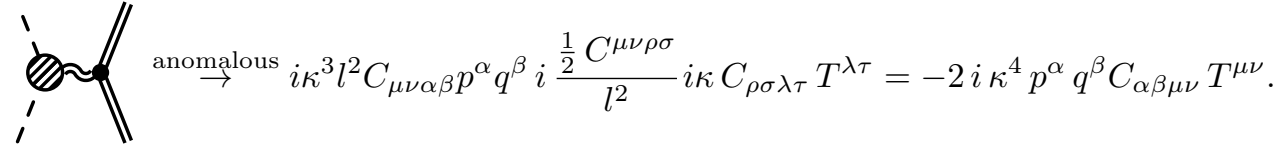

Here $p$ and $q$ are momenta of the ingoing scalar particles. The expression (12) is local, so it corresponds to the following contact interaction:

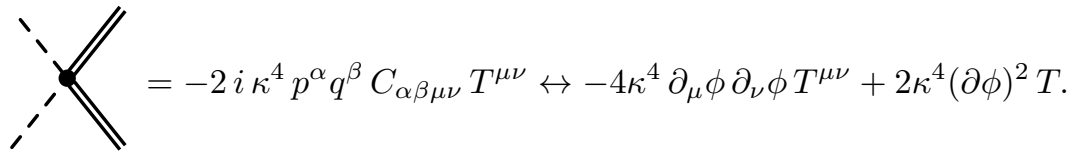

It can be seen clearly that this is a beyond Horndeski interaction (3) with the following parameters:

$$
C(\phi, X)=2 \kappa^{4}(\partial \phi)^{2}, \quad D(\phi, X)=-4 \kappa^{4} .
$$

It should be noted that the amplitude (11) is not the only amplitude that generates beyond Horndeski interactions. However, the other amplitudes experience stronger suppression. Namely, the following amplitude may also generate beyond Horndeski interaction:

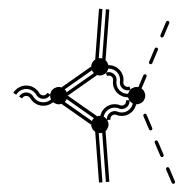

Here double plain lines note matter degrees of freedom. This amplitude may very well generate an interaction between one graviton, two scalars, and two matter degrees of freedom that belong to the beyond Horndeski class:

$$
h_{\mu \nu}(\partial \phi)^{2} T^{\mu \nu} \leftrightarrow C(\phi, X) g_{\mu \nu} T^{\mu \nu}
$$

Firstly, this interaction is suppressed by the factor $\kappa^{5}$, so it does not provide the leading contribution and can be safely neglected for the time being. Secondly, simple dimensional considerations show that this amplitude develops log-dependence of the factorization scale. Although such a dependence of the factorization scale is weak, it does not allow one to consider such a contribution independent from the high energy content of a theory.

The following amplitudes can be treated in a similar way:

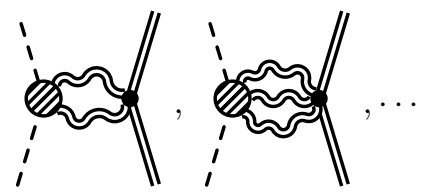

They are suppressed, at very least, by factor $\kappa^{2 N}$, where $N$ is the number of virtual gravitons. Therefore the amplitude (12), considered above, provides the leading contribution.

This proves our original claim, so a certain beyond Horndeski interaction are generated at the one-loop level. The corresponding amplitude (12) is free from ultraviolet divergences and therefore are independent on the high energy content of a quantum gravity model. We discuss this result and its implications in the next Section. 


\section{Discussion and conclusion}

We have shown that a certain beyond Horndeski interaction is universally generated within effective field theory.

Both analytical [26] and non-analytical [21] terms that are independent from the factorization scale $\mu$ which separating low and high energy physics are generated at the loop level. Beyond Horndeski interactions are generated in the same way. Namely, a specific one-loop amplitude (12) contains a part generating the non-minimal kinetic coupling (8) which is independent from the factorization scale. Because of this the amplitude (12) generates a new non-minimal interaction between the scalar field and matter. This interaction belongs to beyond Horndeski class and it does not depend on the factorization scale.

This shows that certain beyond Horndeski interactions are generated in a way similar to anomalous magnetic momentum and to a certain non-minimal kinetic coupling [26].

Because such a beyond Horndeski interaction is generated at the loop level it experience an extremely strong suppression by factor $\kappa^{4} \sim G^{2}$. Moreover, such interactions are local, so they can hardly be found in the low energy (large scale) experiments. Such interactions appear at least at $2 \mathrm{PN}$ (post-Newtonian) order or higher $[43,44]$. Although such effects can be calculated $[45,46,47,48,49,11]$, it would be challenging to efficiently constraint them with the current empirical data.

\section{Acknowledgment}

The work was supported by the Foundation for the Advancement of Theoretical Physics and Mathematics "BASIS".

\section{References}

[1] Gregory Walter Horndeski. Second-order scalar-tensor field equations in a four-dimensional space. Int. J. Theor. Phys., 10:363-384, 1974. doi:10.1007/BF01807638.

[2] Tsutomu Kobayashi, Masahide Yamaguchi, and Jun'ichi Yokoyama. Generalized G-inflation: Inflation with the most general second-order field equations. Prog. Theor. Phys., 126:511529, 2011. arXiv:1105.5723, doi:10.1143/PTP.126.511.

[3] M. Ostrogradsky. Mémoires sur les équations différentielles, relatives au problème des isopérimètres. Mem. Acad. St. Petersbourg, 6(4):385-517, 1850.

[4] Tsutomu Kobayashi. Horndeski theory and beyond: a review. Rept. Prog. Phys., 82(8):086901, 2019. arXiv:1901.07183, doi:10.1088/1361-6633/ab2429.

[5] B. Latosh. Fab Four Effective Field Theory Treatment. Eur. Phys. J., C78(12):991, 2018. arXiv:1812.01881, doi:10.1140/epjc/s10052-018-6470-0.

[6] Alexei A. Starobinsky, Sergey V. Sushkov, and Mikhail S. Volkov. The screening Horndeski cosmologies. JCAP, 1606(06):007, 2016. arXiv:1604.06085, doi:10.1088/1475-7516/ 2016/06/007.

[7] Eugeny Babichev and Christos Charmousis. Dressing a black hole with a time-dependent Galileon. JHEP, 08:106, 2014. arXiv:1312.3204, doi:10.1007/JHEP08(2014)106.

[8] Eugeny Babichev, Christos Charmousis, and Antoine Lehébel. Black holes and stars in Horndeski theory. Class. Quant. Grav., 33(15):154002, 2016. arXiv:1604.06402, doi: $10.1088 / 0264-9381 / 33 / 15 / 154002$.

[9] S. Mironov, V. Rubakov, and V. Volkova. Subluminal cosmological bounce beyond Horndeski. JCAP, 05:024, 2020. arXiv:1910.07019, doi:10.1088/1475-7516/2020/05/024.

[10] V.E. Volkova, S.A. Mironov, and V.A. Rubakov. Cosmological Scenarios with Bounce and Genesis in Horndeski Theory and Beyond. J. Exp. Theor. Phys., 129(4):553-565, 2019. doi:10.1134/S1063776119100236.

[11] P.I. Dyadina, N.A. Avdeev, and S.O. Alexeyev. Horndeski gravity without screening in binary pulsars. Mon. Not. Roy. Astron. Soc., 483(1):947-963, 2019. arXiv:1811.05393, doi:10.1093/mnras/sty3094. 
[12] Gregory W. Horndeski. Lagrange Multipliers and Third Order Scalar-Tensor Field Theories. 8 2016. arXiv: 1608.03212.

[13] Hayato Motohashi, Karim Noui, Teruaki Suyama, Masahide Yamaguchi, and David Langlois. Healthy degenerate theories with higher derivatives. JCAP, 07:033, 2016. arXiv: 1603.09355, doi:10.1088/1475-7516/2016/07/033.

[14] Hayato Motohashi, Teruaki Suyama, and Masahide Yamaguchi. Ghost-free theory with third-order time derivatives. J. Phys. Soc. Jap., 87(6):063401, 2018. arXiv:1711.08125, doi:10.7566/JPSJ .87.063401.

[15] Hayato Motohashi, Teruaki Suyama, and Masahide Yamaguchi. Ghost-free theories with arbitrary higher-order time derivatives. JHEP, 06:133, 2018. arXiv:1804.07990, doi: 10.1007/JHEP06 (2018) 133.

[16] Jacob D. Bekenstein. The Relation between physical and gravitational geometry. Phys. Rev. D, 48:3641-3647, 1993. arXiv:gr-qc/9211017, doi:10.1103/PhysRevD.48.3641.

[17] Miguel Zumalacárregui and Juan García-Bellido. Transforming gravity: from derivative couplings to matter to second-order scalar-tensor theories beyond the Horndeski Lagrangian. Phys. Rev. D, 89:064046, 2014. arXiv:1308.4685, doi:10.1103/PhysRevD.89.064046.

[18] David Langlois, Michele Mancarella, Karim Noui, and Filippo Vernizzi. Effective Description of Higher-Order Scalar-Tensor Theories. JCAP, 05:033, 2017. arXiv:1703.03797, doi: $10.1088 / 1475-7516 / 2017 / 05 / 033$.

[19] Claudia de Rham and Andrew Matas. Ostrogradsky in Theories with Multiple Fields. JCAP, 06:041, 2016. arXiv:1604.08638, doi:10.1088/1475-7516/2016/06/041.

[20] Jibril Ben Achour, David Langlois, and Karim Noui. Degenerate higher order scalar-tensor theories beyond Horndeski and disformal transformations. Phys. Rev. D, 93(12):124005, 2016. arXiv:1602.08398, doi:10.1103/PhysRevD.93.124005.

[21] John F. Donoghue. General relativity as an effective field theory: The leading quantum corrections. Phys. Rev., D50:3874-3888, 1994. arXiv:gr-qc/9405057, doi:10.1103/ PhysRevD.50.3874.

[22] C. P. Burgess. Quantum gravity in everyday life: General relativity as an effective field theory. Living Rev. Rel., 7:5-56, 2004. arXiv:gr-qc/0311082, doi:10.12942/lrr-2004-5.

[23] A. O. Barvinsky and G. A. Vilkovisky. The Generalized Schwinger-Dewitt Technique in Gauge Theories and Quantum Gravity. Phys. Rept., 119:1-74, 1985. doi:10.1016/ 0370-1573(85) 90148-6.

[24] N. E. J Bjerrum-Bohr, John F. Donoghue, and Barry R. Holstein. Quantum gravitational corrections to the nonrelativistic scattering potential of two masses. Phys. Rev., D67:084033, 2003. [Erratum: Phys. Rev.D71,069903(2005)]. arXiv:hep-th/0211072, doi:10.1103/ PhysRevD.71.069903,10.1103/PhysRevD.67.084033.

[25] N. E. J. Bjerrum-Bohr, John F. Donoghue, Barry R. Holstein, Ludovic Plante, and Pierre Vanhove. Light-like Scattering in Quantum Gravity. JHEP, 11:117, 2016. arXiv:1609. 07477, doi:10.1007/JHEP11(2016) 117.

[26] Boris Latosh. One-loop effective scalar-tensor gravity. Eur. Phys. J. C, 80(9):845, 2020. arXiv:2004.00927, doi:10.1140/epjc/s10052-020-8371-2.

[27] Vítor Fernandes Barra, Iosif L. Buchbinder, Jarme Gomes Joaquim, Andreza Rairis Rodrigues, and Ilya L. Shapiro. Renormalization of Yukawa model with sterile scalar in curved spacetime. Eur. Phys. J. C, 79(6):458, 2019. arXiv:1903.11546, doi:10.1140/epjc/ s10052-019-6917-y.

[28] Iosif L. Buchbinder, Andreza Rairis Rodrigues, Eduardo Antonio dos Reis, and Ilya L. Shapiro. Quantum aspects of Yukawa model with scalar and axial scalar fields in curved spacetime. Eur. Phys. J. C, 79(12):1002, 2019. arXiv:1910.01731, doi:10.1140/epjc/ s10052-019-7447-3. 
[29] Christian F. Steinwachs and Alexander Yu. Kamenshchik. One-loop divergences for gravity non-minimally coupled to a multiplet of scalar fields: calculation in the Jordan frame. I. The main results. Phys. Rev. D, 84:024026, 2011. arXiv:1101.5047, doi:10.1103/PhysRevD. 84.024026 .

[30] Lavinia Heisenberg, Johannes Noller, and Jann Zosso. Horndeski under the quantum loupe. JCAP, 10:010, 2020. arXiv:2004.11655, doi:10.1088/1475-7516/2020/10/010.

[31] Ippocratis D. Saltas and Vincenzo Vitagliano. Covariantly Quantum Galileon. Phys. Rev. D, 95(10):105002, 2017. arXiv:1611.07984, doi:10.1103/PhysRevD.95.105002.

[32] A. O. Barvinsky, A. Yu. Kamenshchik, and A. A. Starobinsky. Inflation scenario via the Standard Model Higgs boson and LHC. JCAP, 11:021, 2008. arXiv:0809.2104, doi: $10.1088 / 1475-7516 / 2008 / 11 / 021$.

[33] A. O. Barvinsky, A. Yu. Kamenshchik, C. Kiefer, A. A. Starobinsky, and C. Steinwachs. Asymptotic freedom in inflationary cosmology with a non-minimally coupled Higgs field. JCAP, 12:003, 2009. arXiv:0904.1698, doi:10.1088/1475-7516/2009/12/003.

[34] A. O. Barvinsky, A. Yu. Kamenshchik, C. Kiefer, A. A. Starobinsky, and C. F. Steinwachs. Higgs boson, renormalization group, and naturalness in cosmology. Eur. Phys. J. C, 72:2219, 2012. arXiv:0910.1041, doi:10.1140/epjc/s10052-012-2219-3.

[35] H. Georgi. Effective field theory. Ann. Rev. Nucl. Part. Sci., 43:209-252, 1993. doi: 10.1146/annurev.ns.43.120193.001233.

[36] Andrej Arbuzov and Boris Latosh. Effective Potential of Scalar-Tensor Gravity. Classical and Quantum Gravity, 7 2020. URL: http://iopscience.iop.org/article/10.1088/ 1361-6382/abc572, arXiv:2007.06306, doi:10.1088/1361-6382/abc572.

[37] Bryce S. DeWitt. Quantum Theory of Gravity. 1. The Canonical Theory. Phys. Rev., 160:1113-1148, 1967. [3,93(1987)]. doi:10.1103/PhysRev.160.1113.

[38] Bryce S. DeWitt. Quantum Theory of Gravity. 2. The Manifestly Covariant Theory. Phys. Rev., 162:1195-1239, 1967. [,298(1967)]. doi:10.1103/PhysRev.162.1195.

[39] Bryce S. DeWitt. Quantum Theory of Gravity. 3. Applications of the Covariant Theory. Phys. Rev., 162:1239-1256, 1967. [,307(1967)]. doi:10.1103/PhysRev.162.1239.

[40] L.F. Abbott, Marcus T. Grisaru, and Robert K. Schaefer. The Background Field Method and the S Matrix. Nucl. Phys. B, 229:372-380, 1983. doi:10.1016/0550-3213(83)90337-1.

[41] Arif A. Akhundov, S. Bellucci, and A. Shiekh. Gravitational interaction to one loop in effective quantum gravity. Phys. Lett., B395:16-23, 1997. arXiv:gr-qc/9611018, doi: 10.1016/S0370-2693(96)01694-2.

[42] Marcus T. Grisaru, P. van Nieuwenhuizen, and C.C. Wu. Background Field Method Versus Normal Field Theory in Explicit Examples: One Loop Divergences in S Matrix and Green's Functions for Yang-Mills and Gravitational Fields. Phys. Rev. D, 12:3203, 1975. doi: 10.1103/PhysRevD.12.3203.

[43] Clifford M. Will. The Confrontation between General Relativity and Experiment. Living Rev. Rel., 17:4, 2014. arXiv:1403.7377, doi:10.12942/Irr-2014-4.

[44] C. M. Will. Theory and experiment in gravitational physics. 1993.

[45] Luc Blanchet. Gravitational Radiation from Post-Newtonian Sources and Inspiralling Compact Binaries. Living Rev. Rel., 17:2, 2014. arXiv:1310.1528, doi:10.12942/lrr-2014-2.

[46] Thibault Damour. Gravitational Self Force in a Schwarzschild Background and the Effective One Body Formalism. Phys. Rev. D, 81:024017, 2010. arXiv:0910.5533, doi:10.1103/ PhysRevD . 81.024017.

[47] Luc Blanchet and Thibault Damour. Tail Transported Temporal Correlations in the Dynamics of a Gravitating System. Phys. Rev. D, 37:1410, 1988. doi:10.1103/PhysRevD.37.1410. 
[48] N. Avdeev, P. Dyadina, and S. Labazova. Test of hybrid metric-Palatini $f(R)$-gravity in binary pulsars. J. Exp. Theor. Phys., 131(4):537-547, 2020. arXiv:2009.11156, doi: 10.1134/S1063776120100039.

[49] P.I. Dyadina, S.P. Labazova, and S.O. Alexeyev. Post-Newtonian Limit of Hybrid MetricPalatini $f(R)$-Gravity. J. Exp. Theor. Phys., 129(5):838-848, 2019. doi:10.1134/ S1063776119110025. 